\title{
An Extended General Location Model for Causal Inferences from Data Subject to Noncompliance and Missing Values
}

\author{
Yahong Peng, ${ }^{1, *}$ Roderick J. A. Little, ${ }^{2}$ and Trivellore E. Raghunathan ${ }^{2}$ \\ ${ }^{1}$ Merck Research Laboratories, BL 3-2, West Point, Pennsylvania 19486, U.S.A. \\ ${ }^{2}$ Biostatistics Department, SPH, University of Michigan, Ann Arbor, Michigan 48105, U.S.A. \\ *email: yahong_peng@merck.com
}

\begin{abstract}
Summary. Noncompliance is a common problem in experiments involving randomized assignment of treatments, and standard analyses based on intention-to-treat or treatment received have limitations. An attractive alternative is to estimate the Complier-Average Causal Effect (CACE), which is the average treatment effect for the subpopulation of subjects who would comply under either treatment (Angrist, Imbens, and Rubin, 1996, Journal of American Statistical Association 91, 444-472). We propose an extended general location model to estimate the CACE from data with noncompliance and missing data in the outcome and in baseline covariates. Models for both continuous and categorical outcomes and ignorable and latent ignorable (Frangakis and Rubin, 1999, Biometrika 86, 365-379) missing-data mechanisms are developed. Inferences for the models are based on the EM algorithm and Bayesian MCMC methods. We present results from simulations that investigate sensitivity to model assumptions and the influence of missing-data mechanism. We also apply the method to the data from a job search intervention for unemployed workers.
\end{abstract}

KEY worDs: Causal inference; EM algorithm; General location model; Missing data; Noncompliance.

\section{Introduction}

Inferences for treatment effects are relatively straightforward in randomized trials with perfect compliance with the assigned treatments. However, for scientific experiments involving human participants, noncompliance and partial compliance are very common in practice. Compliance often varies according to participant characteristics, and may be associated with effects of the treatments. For example, in a clinical trial to test the efficacy of a new drug, severe side effects may result in the lack of compliance. Misleading conclusions are likely to result if compliance information is ignored in such settings. Missing values in the outcome or the covariates further complicate the data analysis. This article concerns methods for analyzing data from a clinical trial with all-or-nothing compliance and missing values in the outcomes and covariates.

Intent-to-treat and as-treated methods provide simple estimates of treatment effects and are widely applied in practice, but both these methods have limitations. The intent-to-treat analysis compares the outcome distributions of the treatments as randomized, ignoring compliance information. It provides a valid estimate of the effect of the treatment assignment, but a potentially biased estimate of the effect of the treatment itself, which is often of more interest. The as-treated analysis compares the outcome distributions between the treatments actually received. It focuses more directly on the treatment effect itself, but is subject to selection bias when there is noncompliance, since the randomization is compromised. Alternative analysis methods for noncompliance in clinical trials have received considerable attention recently, and largely focus on two different problems. One concerns inference for the average treatment effect that would have been observed if participants had all fully complied with the treatment regimen, assuming that obstacles such as side effects of drugs could be overcome (Robins and Tsiatis, 1991; Mark and Robins, 1993; Robins, 1998). The second problem is to estimate the Complier-Average Causal Effect (CACE), defined by AIR as the average causal effect for the subpopulation of subjects who would comply with either of the treatments if assigned to them, using the randomization indicator as an instrumental variable (Bloom, 1984; Sommer and Zeger, 1991; Goetghebeur and Shapiro, 1996; Imbens and Rubin, 1996, 1997; Baker, 1998; Frangakis and Rubin, 1999, 2002; Yau and Little, 2001). We call compliance under both treatments "principal compliance" to distinguish it from "observed compliance," since it is a form of principal stratification (Frangakis and Rubin, 2002) for correct causal inference with posttreatment variables. Unlike observed compliance, principal compliance is incompletely observed since compliance under the treatment not assigned is unknown. Building on this work, we propose an extended general location (EGL) model for the CACE for data with noncompliance and missing values. Our model extends methods in Little and Yau (1998) to accommodate discrete as well as continuous outcomes, missing values in baseline covariates as well as in outcomes, and two alternative assumptions about the compliance and missing-data mechanisms.

We motivate and illustrate our model using data from the JOBS II (Vinokur, Price, and Schul, 1995), a randomized trial at the University of Michigan to test a job search intervention for unemployed workers. In this trial unemployed 
individuals were randomly assigned to a control treatment consisting of a booklet describing job search methods and tips, or an experimental treatment group consisting of the same booklet and five 4-hour job search seminars. Follow-up questionnaires were mailed periodically to all the participants, and measures of outcomes such as depression, financial strain, and re-employment were obtained. We focus here on a binary outcome, re-employment at a fixed date after the intervention, but we also consider models for a continuous outcome. The JOBS II data have missing values, and only about one half of the individuals assigned to the experimental treatment complied, in the sense that they attended at least one of the seminars. Since "compliance" with the control treatment is not an issue here, it is assumed that individuals would comply with the control treatment, but may or may not comply with the experimental treatment. Thus compliance (in the CACE sense) is known for participants assigned to the experimental group, but is not known for participants assigned to the control group, since their participation if assigned the intervention is not observed.

The primary outcome in application is thus not medical, but our methods provide alternatives to existing approaches of analysis in any clinical trial with the following characteristics: (a) an active treatment is compared with a control treatment; (b) there is noncompliance with the active treatment, and partial compliance effects are either not present or ignored for simplicity; (c) interest concerns "as-treated" effects, specifically the treatment effect in the subgroup of individuals who would comply with both control and active treatment; and (d) there are missing data in the covariates or outcome. The main limiting feature is that partial compliance effects are ignored, since in treatments involving administration of drugs partial compliance is common. Thus extensions of our methods to handle this situation would be beneficial.

Section 2 presents our EGL model for inferences about the CACE, and inferences based on the EM algorithm and Bayesian MCMC methods. An alternative approach based on instrumental variables is given in Section 2.4. Section 3 presents simulation studies that compare our likelihood-based approach with instrumental variable methods for causal inference (Bloom, 1984; Heckman and Hotz, 1989; Sommer and Zeger, 1991; Zeger and Liang, 1991). In Section 4 we apply our methods to JOBS II data. Section 5 contains conclusions and discussion.

\section{Extended General Location Model}

\subsection{The Complete-Data Model}

We define the following variables for each participant $i$ in a randomized clinical trial:

$x_{i}=\left(x_{i 1}, \ldots, x_{i p}\right)^{\mathrm{T}}=p$ continuous baseline covariates. While these might be treated as fixed in a completedata analysis, we assign them a normal distribution to allow cases with missing values in these variables to be included in the analysis. Transformations may be needed to improve the normality assumption.

$b_{i}=$ discrete baseline covariates. For simplicity, we assume $b_{i}$ is scalar and binary, although the extension to more than two levels is immediate. $r_{i}=$ randomization indicator for the treatment assigned. In the JOBS II application, $r_{i}=1$ for the intervention, $r_{i}=0$ for the control treatment.

$y_{i}=$ an outcome variable, which we initially assume is continuous and normal. Extensions to vector outcomes are outlined in Section 5.

$c_{i}=$ principal compliance indicator, taking the value 1 if participant $i$ would comply with either treatment, and 0 otherwise. As noted above, in the JOBS II setting $c_{i}$ is observed for participants assigned to the experimental group, taking the value 1 for individuals who attended the seminars and 0 otherwise. There was a small amount of partial compliance in JOBS II in that a few individuals attended a subset of the seminars but not all of them; for the purposes of our analysis these participants are treated as compliers. For individuals assigned to the control group, $c_{i}$ is missing since we do not know whether they would have attended the seminars if they had been assigned to the experimental group.

We write $[A \mid B]$ to denote the distribution of $A$ given $B, N$ for a univariate normal and $N_{p}$ for a $p$-variate normal distribution. We assume observations are independently distributed given the randomization indicator $r_{i}$. Independence is violated when potential outcomes for each unit are related to the treatments assigned and the treatments actually received by the other units. Thus we make the stable unit treatment value assumption (SUTVA) that potential outcomes for each unit are unrelated to the treatments assigned and the treatments actually received by the other units (Angrist, Imbens, and Rubin, 1996). While this assumption is almost universally invoked, it is somewhat suspect in the JOBS II setting, since the experimental treatment takes place in a group setting. For a Bayesian approach to modeling noncompliance under group randomization, see Frangakis, Rubin, and Zhou (2002).

We factor the joint distribution $\left[y_{i}, x_{i}, c_{i}, b_{i} \mid r_{i}\right]$ as $\left[y_{i}, x_{i}, c_{i}, b_{i} \mid r_{i}\right]=\left[c_{i}, b_{i} \mid r_{i}\right] \cdot\left[x_{i} \mid c_{i}, b_{i}, r_{i}\right] \cdot\left[y_{i} \mid x_{i}, c_{i}, b_{i}, r_{i}\right]$. We then model the components of this factorization as follows:

$$
\begin{aligned}
{\left[c_{i}, b_{i} \mid r_{i}\right] } & \sim \operatorname{Multinomial}(\alpha), \\
{\left[x_{i} \mid c_{i}=c, b_{i}=b, r_{i}=r\right] } & \sim N_{p}\left(\mu_{c b}, \Sigma\right), \\
{\left[y_{i} \mid x_{i}, c_{i}, b_{i}, r_{i}\right] } & \sim N\left\{g\left(x_{i}, c_{i}, b_{i}, r_{i} ; \beta\right), \tau^{2}\right\},
\end{aligned}
$$

where

$$
\begin{aligned}
g\left(x_{i}, c_{i}, b_{i}, r_{i} ; \beta\right)= & \beta_{0}+\beta_{C} c_{i}+\beta_{C R} c_{i} r_{i}+\beta_{B} b_{i}+\beta_{X} x_{i} \\
& +\beta_{B C} b_{i} c_{i}+\beta_{X C} x_{i} c_{i} \\
& +\beta_{B C R} b_{i} c_{i} r_{i}+\beta_{X C R} x_{i} c_{i} r_{i} .
\end{aligned}
$$

The parameters of the model are thus: $\theta=\left(\alpha, \mu_{c b}, \Sigma, \beta, \tau^{2}\right)$, where $\mu_{c b}$ is a $(p \times 1)$ vector, $\Sigma=\left(\sigma_{i j}\right)$ is a $(p \times p)$ matrix, and $\beta^{\mathrm{T}}=\left(\beta_{0}, \beta_{C}, \beta_{C R}, \beta_{B}, \beta_{X}, \beta_{B C}, \beta_{X C}, \beta_{B C R}, \beta_{X C R}\right)$. The parameterization of this model embodies the following important assumptions, described in more detail in AIR:

(1) Parameters $\left(\alpha, \mu_{c b}, \Sigma\right)$ do not depend on $r_{i}$ because that randomization of treatments ensures that $r_{i}$ is independent of $c_{i}, b_{i}, x_{i}$. 
(2) The mean structure in (4) omits the effects of $r_{i}, b_{i} r_{i}$, and $x_{i} r_{i}$, because we assume that for noncompliers $\left(c_{i}=0\right)$ the effect of treatment is the same whether subject $i$ is randomized to control (and has no access to the treatment) or randomized to the treatment (and fails to comply). This is the crucial exclusion restriction (ER) assumption discussed in AIR. Equation (4) implies that the mean of $y_{i}$ for the noncompliers with baseline covariates $\left(b_{i}=b, x_{i}=x\right)$ is $g(x, 0, b, r ; \beta)=\beta_{0}+\beta_{B} b+$ $\beta_{X} x$, and the CACE for compliers with covariates $\left(b_{i}=b, x_{i}=x\right)$ is then $\operatorname{CACE}(b, x)=g(x, 1, b, 1 ; \beta)-$ $g(x, 1, b, 0 ; \beta)=\beta_{C R}+\beta_{B C R} b+\beta_{X C R} x$. An overall CACE can be defined by averaging over the distribution $p(b, x \mid c=1)$ of $B$ and $X$ for compliers:

$$
\mathrm{CACE}=\int_{b, x} \operatorname{CACE}(b, x) p(b, x \mid c=1) d x d b .
$$

We call this an extended general location (EGL) model because the distribution of the continuous variables $\left[y_{i}, x_{i} \mid c_{i}, b_{i}, r_{i}\right]$ is normal with a covariance matrix that depends on the discrete variables $\left(c_{i}, b_{i}, r_{i}\right)$, rather than being a constant as in the standard general location model (Olkin and Tate, 1961; Little and Rubin, 2002, Section 14.2). Restrictions can be imposed on the EGL model, either by assuming a log-linear model for $\left[c_{i}, b_{i} \mid r_{i}\right]$, or by constraining the means $\mu_{c b}$ of the normal model for $\left[x_{i} \mid c_{i}, b_{i}, r_{i}\right]$. Restrictions are particularly helpful in reducing the number of parameters when the sample size is small. The distinctness of the parameters of the models for $\left[c_{i}, b_{i} \mid r_{i}\right]$ and for $\left[x_{i} \mid c_{i}, b_{i}, r_{i}\right]$ is preserved, so estimation procedures are not radically different than those for unrestricted models. With appropriate restrictions on the model parameters $\left(\alpha, \mu_{c b}, \Sigma\right)$ of $\left[x_{i}, c_{i}, b_{i} \mid r_{i}\right]$, the EGL model yields $\left[c_{i} \mid x_{i}, b_{i}, r_{i}\right]$ similar to those in Little and Yau (1998) after reparameterization (Peng, 2001).

Equation (3) of the EGL model can also be adapted to a binary outcome, say $y_{i}^{*}$. We assume there is an underlying latent continuous variable $y_{i}$ for $y_{i}^{*}$, where $y_{i}^{*}=I\left(y_{i}>0\right)$. The distribution $\left[y_{i} \mid x_{i}, c_{i}, b_{i}, r_{i}\right]$ is given by equation (3) with $\tau^{2}=1$. This implies a probit model for $y_{i}^{*}$, namely,

$$
\operatorname{Pr}\left(y_{i}^{*}=1 \mid x_{i}, c_{i}, b_{i}, r_{i}\right)=\Phi\left\{g\left(x_{i}, c_{i}, b_{i}, r_{i} ; \beta\right)\right\},
$$

where $\Phi(\cdot)$ is the standard normal cumulative probability function. When the outcome of interest is binary, $\Phi\{g(x, 1, b, 1 ; \beta)\}-\Phi\{g(x, 1, b, 0 ; \beta)\}$ is the CACE for subjects with covariates $\left(b_{i}=b, x_{i}=x\right)$, and the overall CACE can be calculated as in equation (5). The probit model (6) for $\left[y_{i}^{*} \mid x_{i}, c_{i}, b_{i}, r_{i}\right]$ is proposed rather than the more common logit model because it is more convenient for computation, as discussed in Section 2.3.

\subsection{Missing-Data Mechanisms}

We now suppose there are missing values of $\left(x_{i}, b_{i}, y_{i}\right)$ in the data set, in addition to the missing values of $c_{i}$ in the control group. The validity of methods for handling the missing data depends on assumptions about mechanisms that create missing values. For subject $i$, let $w_{i}=\left(x_{i}, b_{i}, y_{i}\right)$ denote the set of values if they were fully observed, and write $w_{o b s, i}$ for the observed values of $w_{i}$ and $w_{m i s, i}$ for the missing values. Also, let $m_{i}$ be the vector of missing-data indicators for $w_{i}$, with entries 1 if the corresponding entries of $w_{i}$ are missing and 0 otherwise. The missing-data mechanism for $w_{i}$ is specified via a distribution $\operatorname{Pr}\left(m_{i} \mid w_{i}, c_{i}, r_{i} ; \phi\right)$ for $m_{i}$ given $w_{i}$ and compliance and randomization indicators $\left(c_{i}, r_{i}\right)$, indexed by unknown parameters $\phi$. We consider two alternative assumptions about the missing-data mechanism.

Ignorable missing-data mechanism (IMD). The missing-data mechanism of $w_{i}$ is said to be ignorable if the data are missing at random (MAR; Rubin, 1976; Little and Rubin, 2002), that is: $\operatorname{Pr}\left(m_{i} \mid w_{i}, c_{i}, r_{i}=1 ; \phi\right)=\operatorname{Pr}\left(m_{i} \mid w_{o b s, i}, c_{i}, r_{i}=1 ; \phi\right)$, for all $w_{m i s, i}, \operatorname{Pr}\left(m_{i} \mid w_{i}, c_{i}, r_{i}=0 ; \phi\right)=\operatorname{Pr}\left(m_{i} \mid w_{o b s, i}, r_{i}=0 ; \phi\right)$, for all $w_{m i s, i}, c_{i}$, and the parameters $\phi$ are distinct from the parameters $\theta$ of the complete-data model. ML and Bayes estimation under this assumption does not require a term for the missing-data mechanism to be included in the likelihood.

Note that the mechanism is allowed to depend on $c_{i}$ for experimental subjects $\left(r_{i}=1\right)$ since $c_{i}$ is observed for these subjects, but is not allowed to depend on $c_{i}$ for control subjects $\left(r_{i}=0\right)$ since $c_{i}$ is missing for these subjects. The latter may not be realistic, since the probability of nonresponse for noncompliers may be systematically higher than that for compliers, regardless of treatment. To handle such cases, Frangakis and Rubin (1999) propose an instrumental variable estimator for an alternative missing-data mechanism, which they call latent ignorability.

Latent ignorable missing-data mechanism (LIMD). The missing-data mechanism of $w_{i}$ is said to be latent ignorable if the data are latent MAR (LMAR) within each level of the latent compliance status, that is: $\operatorname{Pr}\left(m_{i} \mid w_{i}, c_{i}, r_{i}=1 ; \phi\right)=$ $\operatorname{Pr}\left(m_{i} \mid w_{o b s, i}, c_{i}, r_{i}=1 ; \phi\right)$, for all $w_{m i s, i}, \operatorname{Pr}\left(m_{i} \mid w_{i}, c_{i}, r_{i}=0\right.$; $\phi)=\operatorname{Pr}\left(m_{i} \mid w_{\text {obs }, i}, c_{i}, r_{i}=0 ; \phi\right)$, for all $w_{m i s, i}$, and the parameters $\phi$ are distinct from the parameters of the completedata model (see also Barnard et al. [2002]). Note that LMAR is weaker than MAR since the mechanism is allowed to depend on $c_{i}$ for control as well as experimental subjects. This missing-data mechanism is in general nonignorable, because $c_{i}$ is unknown in the control group.

As LIMD is weaker than IMD, a stronger exclusion restriction assumption is needed to identify the parameters when the complete-data model is saturated, and inference may be vulnerable to misspecification when the complete-data model is unsaturated. Hence, in our LIMD models we replace the ER assumption above by the following stronger assumption.

Compound exclusion restriction (CER). The joint distribution $\left(y_{i}, m_{i}\right)$ for a noncomplier randomized to the control group is the same as the distribution $\left(y_{i}, m_{i}\right)$ for a noncomplier randomized to the treatment group.

Our LIMD model involves a particular form of LIMD where missingness of $y_{i}$ is allowed to depend on missing values of $c_{i}$, but missingness of $\left(x_{i}, b_{i}\right)$ is not. Accordingly, we write $m_{i}=$ $\left(m_{i}^{Y}, m_{i}^{X B}\right)$, where $m_{i}^{Y}$ is the missing-data indicator for $y_{i}$ and $m_{i}^{X B}$ is the vector of missing-data indicators for $x_{i}$ and $b_{i}$, and assume for all $w_{m i s, i}, c_{i}, \operatorname{Pr}\left(m_{i}^{Y}, m_{i}^{X B} \mid w_{i}, c_{i}, r_{i}=0 ; \phi\right)$ $=\operatorname{Pr}\left(m_{i}^{Y} \mid w_{o b s, i}, c_{i}, r_{i}=0 ; \phi_{1}\right) \cdot \operatorname{Pr}\left(m_{i}^{X B} \mid m_{i}^{Y}, w_{o b s, i}, r_{i}=\right.$ $\left.0, \phi_{2}\right)$, where $\phi=\left(\phi_{1}, \phi_{2}\right)$ are distinct from $\theta$. By a straightforward extension of Rubin's (1976) theory, only the component $m_{i}^{Y}$ of $m_{i}$ distribution needs to be modeled in this case. We then factor $\left[y_{i}, x_{i}, c_{i}, b_{i}, m_{i}^{Y} \mid r_{i}\right]=\left[c_{i}, b_{i} \mid r_{i}\right] \cdot\left[x_{i} \mid c_{i}, b_{i}, r_{i}\right]$. $\left[m_{i}^{Y} \mid x_{i}, c_{i}, b_{i}, r_{i}\right] \cdot\left[y_{i} \mid m_{i}^{Y}, x_{i}, c_{i}, b_{i}, r_{i}\right]$, where (a) $\left[y_{i} \mid m_{i}^{Y}\right.$ $\left.x_{i}, c_{i}, b_{i}, r_{i}\right]=\left[y_{i} \mid x_{i}, c_{i}, b_{i}, r_{i}\right]$ because of the latent ignorability assumption; (b) $\left[c_{i}, b_{i} \mid r_{i}\right],\left[x_{i} \mid c_{i}, b_{i}, r_{i}\right]$, and $\left[y_{i} \mid x_{i}, c_{i}, b_{i}, r_{i}\right]$ 
can be modeled as in equations (1-3); and (c) $\left[m_{i}^{Y} \mid x_{i}=x\right.$, $\left.c_{i}=c, b_{i}=b, r_{i}=r\right] \sim \operatorname{Bernoulli}\left(\phi_{x c b r}\right)$. To model the latter distribution, we assume there is an underlying continuous variable $x_{i}^{M}$ for $m_{i}^{Y}$, such that $m_{i}^{Y}=I\left(x_{i}^{M}>0\right)$. We then assume that, given $\left(x_{i}, c_{i}, b_{i}, r_{i}\right), x_{i}^{M}$ follows a normal distribution with mean $g\left(x_{i}, c_{i}, b_{i}, r_{i} ; \nu\right)$ and variance 1 , where $g(\cdot)$ is defined in (4) with regression coefficients $\beta$ replaced by $\nu$. This implies a probit model for $\left[m_{i}^{Y} \mid x_{i}, c_{i}, b_{i}, r_{i}\right]$. The CER assumption implies that the mean structure of $x_{i}^{M}$ does not include the effects of $r_{i}, x_{i} r_{i}, b_{i} r_{i}$, that is: $E\left(x_{i}^{M} \mid x_{i}, c_{i}=0\right.$, $\left.b_{i}, r_{i}\right)=\nu_{0}+\nu_{D} b_{i}+\nu_{X} x_{i}$. This is also an extended general location model for $\left[y_{i}, x_{i}^{M}, x_{i}, c_{i}, b_{i} \mid r_{i}\right]$. The extension for binary $y_{i}^{*}$ is carried out in the same way as before.

\subsection{Maximum Likelihood and Bayes Estimation}

Under IMD and ER, maximum likelihood (ML) estimates for the parameters of our EGL model can be obtained via the EM algorithm (Dempster, Laird, and Rubin, 1977), and is similar to EM for the general location model (Little and Rubin, 2002). We outline the EM algorithm for the unrestricted extended general location model of Section 3.1. Let $d_{i}=\left(d_{i 1}, d_{i 2}\right.$, $\left.d_{i 3}, d_{i 4}\right)$ be the vector of indicators for the discrete variables $\left(c_{i}, b_{i}\right)$, with $d_{i 1}=I\left(c_{i}=b_{i}=0\right), d_{i 2}=I\left(c_{i}=1, b_{i}=0\right)$, $d_{i 3}=I\left(c_{i}=0, b_{i}=1\right), d_{i 4}=I\left(c_{i}=b_{i}=1\right)$, with corresponding probabilities $\alpha=\left(\alpha_{1}, \alpha_{2}, \alpha_{3}, a_{4}\right)$. The complete-data likelihood can be written as

$$
\begin{aligned}
L_{c o m}(\theta) \propto \prod_{i}[ & \left\{\prod_{j=1}^{4}\left(\alpha_{j}^{d_{i j}}\right)\right\} \cdot|\Sigma|^{-(p / 2)} \\
& \times \exp \left\{-\frac{1}{2}\left(x_{i}-\mu_{c_{i} b_{i}}\right)^{\mathrm{T}} \Sigma^{-1}\left(x_{i}-\mu_{c_{i} b_{i}}\right)\right\} \\
& \left.\cdot \frac{1}{\tau} \exp \left\{-\frac{1}{2 \tau^{2}}\left(y_{i}-g\left(x_{i}, c_{i}, b_{i}, r_{i} ; \beta\right)\right)^{2}\right\}\right] .
\end{aligned}
$$

The logarithm of this likelihood is linear in complete-data sufficient statistics $\sum_{i} r_{i}, \quad \sum_{i} d_{i j}, \quad \sum_{i} d_{i j} x_{i}, \quad \sum_{i} d_{i j} y_{i}$, $\sum_{i} d_{i j} x_{i} x_{i}^{\mathrm{T}}, \sum_{i} d_{i j} x_{i} y_{i}, \quad \sum_{i} d_{i j} y_{i}^{2}, \quad \sum_{i} r_{i} d_{i j}, \quad \sum_{i} r_{i} d_{i j} x_{i}$, $\sum_{i} r_{i} d_{i j} y_{i}, \quad \sum_{i} r_{i} d_{i j} x_{i} x_{i}^{\mathrm{T}}, \quad \sum_{i} r_{i} d_{i j} x_{i} y_{i}, \quad$ and $\quad \sum_{i}^{i} r_{i} d_{i j} y_{i}^{2}$, for $j=1, \ldots, 4$. The maximization step (M-step) of EM calculates the parameter estimates that maximize the complete-data likelihood given the sufficient statistics calculated from the current E-step, and is straightforward. Specifically, $\alpha_{j}$ is estimated by the expected count in the corresponding cell formed by the discrete variables, $\mu_{c b}$ by the sample mean of $X$ with subjects with $\left(c_{i}=c, b_{i}=b\right), \Sigma$ by the pooled within-cell covariance matrix of $X$, and $\beta$ and $\tau^{2}$ by the regression $y_{i}$ on the components of $\left(x_{i}, c_{i}, b_{i}, r_{i}\right)$ included in the model.

The expectation step (E-step) of EM calculates the conditional expectations of the complete-data sufficient statistics, given the observed data and the current estimates of the parameters from the M-step. It is easier to calculate the conditional expectations for the sufficient statistics $\sum_{i} d_{i}$ first, and then calculate the conditional expectations of the sufficient statistics that involve continuous variables $\left(x_{i}, y_{i}\right)$ and the interaction terms between the discrete and continuous variables. Calculations of the conditional expectations of the sufficient statistics that contain continuous variables $\left(x_{i}, y_{i}\right)$ involve the parameters of conditional distributions of the multivariate normal. They are easily computed by the SWEEP opera- tor (e.g., Little and Rubin, 2002). The E-step can be carried out by missing-data pattern to avoid unnecessary SWEEP computations.

The initial values for EM cannot be computed using data from the set of complete cases, because the values of $c_{i}$ are completely unknown in the control group. To avoid this, we first estimate $\alpha$ by using only the data from the treatment group subjects, and then impute values of $c_{i}$ for the control group subjects by the estimated $\alpha$. We then calculate the sufficient statistics, ignoring those cases with missing values in $\left(x_{i}, b_{i}, y_{i}\right)$, and do an M-step based on the imputed sufficient statistics to obtain initial estimates for $\beta$ 's. The method of moments can also be used to provide initial estimates after imputing the compliance status for control group subjects.

The observed data likelihood is readily computed from byproducts of the E-step with each iteration, providing a check of the computations. In addition, we can use the observed data likelihood to perform likelihood-ratio tests for choosing between models.

For Bayesian inferences, we assume $\alpha,(\mu, \Sigma)$, and $\left(\beta, \tau^{2}\right)$ are a priori independent with conventional noninformative priors, $[\alpha] \propto \prod_{j=1}^{4} \alpha_{j}^{-0.5},[\mu, \Sigma] \propto|\Sigma| \cdot \exp \{-(p+1) / 2\}$, and $\left[\beta, \tau^{2}\right] \propto 1 / \tau^{2}$. These distributions yield a complete-data posterior distribution that is Dirichlet for $\alpha$, inverse-Wishart for $\Sigma$ and multivariate normal for $\mu$ given $\Sigma$, scaled inv- $\chi^{2}$ for $\tau^{2}$ and multivariate normal distribution for $\beta$ given $\tau^{2}$ : $[\alpha \mid$ Data $] \propto \prod_{j=1}^{4}\left\{\alpha_{j} \exp \left(\sum_{i} d_{i j}-0.5\right)\right\},[\Sigma \mid$ Data, $\alpha] \sim$ $W^{-1}\left(N-4, N \hat{\Sigma}^{-1}\right),\left[\mu_{c b} \mid\right.$ Data, $\left.\alpha, \Sigma\right] \sim N\left\{\hat{\mu}_{c b}, \Sigma / \sum_{i} I\left(c_{i}=c\right.\right.$, $\left.\left.b_{i}=b\right)\right\},\left[\tau^{2} \mid\right.$ Data, $\left.\alpha, \mu, \Sigma\right] \sim \hat{\tau}^{2} / \chi_{N-k}^{2},\left[\beta \mid\right.$ Data, $\left.\alpha, \Sigma, \mu, \tau^{2}\right] \sim$ $N\left\{\hat{\beta}, \tau^{2}\left(Z^{T} Z\right)^{-1}\right\}$, where $Z$ is the design matrix of the regression of $y_{i}$ on $x_{i}, c_{i}, b_{i}, r_{i},\left(\hat{\mu}, \hat{\Sigma}, \hat{\beta}, \hat{\tau}^{2}\right)$ are the ML estimates for model parameters $\left(\mu, \Sigma, \beta, \tau^{2}\right)$ based upon the complete-data likelihood. The Gibbs sampler algorithm iterates between the P-step and the I-step. The th P-step of the Gibbs sampler algorithm simulates random draws, $\left\{\alpha^{(t)}, \mu^{(t)}, \Sigma^{(t)}, \beta^{(t)}, \tau^{2(t)}\right\}$, for the model parameters based on the complete-data posterior distribution with missing data filled in from the previous I-step. The I-step is very similar to the E-step of EM. Instead of calculating the conditional expectations of missing values in terms of the sufficient statistics, random draws for the missing values in the data set are generated from their predictive distribution, using current draws of the parameters from the previous P-step. When the observable outcome of interest is discrete, $\tau^{2}=1$, and drawing values of $y_{i}$ involves simulating random numbers from truncated normal distributions, since we only observe the sign of $y_{i}$.

We used the method of Gelman and Rubin (1992) to assess convergence of the Gibbs sequences. To obtain reasonably overdispersed random numbers for the starting points for the Gibbs draws, $m$ bootstrap samples were generated from the original data set with a sample size of half the size of the original data set. For each of the bootstrap samples, ML estimates are obtained via the EM algorithm and used as the starting values for the Gibbs algorithm.

Quite straightforward modifications of these algorithms yield ML and Bayes estimates for the LIMD/CER model of the previous section. For the M-step of EM, everything remains the same except that we need an additional step for estimating $\nu$. Because the regression models involving $\nu$ and $\beta$ have the same structure, the M-step for $\nu$ is the same as 
that for $\beta$, with $x_{i}^{M}$ replacing $y_{i}$ in expressions involving these terms. For the E-step, conditional expectations also need to be computed for complete-data sufficient statistics associated with the model for $x_{i}^{M}$, namely: $\left\{\sum_{i} x_{i}^{M}, \sum_{i}\left(d_{i j} x_{i}^{M}\right)\right.$, $\sum_{i}\left(x_{i}^{M} x_{i}\right), \quad \sum_{i}\left(d_{i j} x_{i}^{M} x_{i}\right), \quad \sum_{i}\left(r_{i} x_{i}^{M}\right), \quad \sum_{i}\left(r_{i} d_{i j} x_{i}^{M}\right)$, $\left.\sum_{i}^{i}\left(r_{i} x_{i}^{M} x_{i}\right), \sum_{i}\left(r_{i} d_{i j} x_{i}^{M} x_{i}\right)\right\}, j=1, \ldots, 4$.

For Bayesian inference we assume a flat prior for $\nu, p(\nu) \propto$ constant, and the priors for the other parameters in the IMD model. The P-step is as before except that we need additional draws for $\nu$ from its posterior distribution $N\left\{\hat{\nu},\left(Z^{\prime} Z\right)^{-1}\right\}$, where $\hat{\nu}$ is the ML estimate of $\nu$ obtained via the EM algorithm based on the imputed data set. For the I-step, we draw the missing values in the discrete variables $\left(c_{i}, b_{i}\right)$ first, then draw the missing continuous variables in $\left(x_{i}, x_{i}^{M}, y_{i}\right)$ based on the corresponding predictive distribution given the current draws for the missing discrete variable and the observed data.

\subsection{Bloom's Method and Extensions}

We conducted simulation studies to compare the modelbased methods in Section 3 with Bloom's (1984) instrumental variable (IV) approach for continuous outcomes with noncompliance, and extensions discussed below.

With complete data and no covariates, the standard IV estimator of the CACE is $\hat{\delta}_{I V}=\left(\bar{y}_{1}-\bar{y}_{0}\right) / \hat{\pi}_{c}$, where $\bar{y}_{j}$ is the mean outcomes of all subjects with $r_{i}=j$, and $\hat{\pi}_{c}$ is the proportion of compliers in the experimental group, $r_{i}=1$. When baseline covariates $\left(x_{i}, b_{i}\right)$ are available, Bloom's (1984) adjusted estimate is $\hat{\delta}_{I V n}=\hat{\beta}_{P}+\left\{\left(1-\hat{\pi}_{c}\right) / \hat{\pi}_{c}\right\} \cdot \hat{\beta}_{N S}$, where $\hat{\beta}_{P}$ and $\hat{\beta}_{N S}$ are the least square estimates from the regression model $y_{i}=\beta_{0}+\beta_{P}\left(r_{i} c_{i}\right)+\beta_{N S} r_{i}\left(1-c_{i}\right)+\beta_{B} b_{i}+$ $\beta_{X} x_{i}+\epsilon_{i}$. The estimator reduces to the standard IV estimator in the absence of baseline covariates. We call $\hat{\delta}_{I V n}$ the naive instrumental variable (IVn) estimator, as it implicitly makes the strong assumption that the compliance rate $\pi_{c}$ is constant for different covariates $(x, b)$, i.e., the compliance is independent of $(x, b)$. A better estimator can be obtained by first estimating the CACE $\delta\left(x_{i}, b_{i}\right)$ for compliers with covariates $\left(x_{i}, b_{i}\right)$ as, $\hat{\delta}\left(x_{i}, b_{i}\right)=\hat{\beta}_{P}+\left\{\left(1-\hat{\pi}_{c i}\right) / \hat{\pi}_{c i}\right\} \cdot \hat{\beta}_{N S}$, where $\hat{\pi}_{c i}$ is the estimated compliance rate for subjects with covariates $\left(x_{i}, b_{i}\right)$, and then estimating the overall causal effect $\delta$ as follows, $\hat{\delta}_{I V}=\int_{X, B} \hat{\delta}(x, b) p(x, b) d x d b$. The IV method in the simulation is this estimator applied to the complete cases. We also considered two IV estimators for the situation where the missing-data mechanism for $y_{i}$ is latent ignorable. The naive latent IV estimate is $\hat{\delta}_{I V L n}=\hat{\beta}_{P}+\left[\left\{\left(1-\hat{\pi}_{c}\right)\left(1-\hat{\phi}_{01}\right)\right\} /\right.$ $\left.\left\{\left(1-\hat{\phi}_{0}\right)-\left(1-\hat{\pi}_{c}\right)\left(1-\hat{\phi}_{01}\right)\right\}\right] \cdot \hat{\beta}_{N S}$, where $\hat{\phi}_{01}$ and $\hat{\phi}_{0}$ are the nonresponse rates in the treatment noncomplier and control groups, respectively. With a homogeneous compliance rate, this is a consistent estimate of the CACE given by Frangakis and Rubin (1999) for LIMD. The IVL estimator with heterogeneous compliance rate $\hat{\delta}_{I V L}$ is obtained by replacing $\hat{\pi}_{c}$ in this expression by a predicted compliance rate $\hat{\pi}_{c i}$ that depends on the covariates $\left(x_{i}, b_{i}\right)$, and then integrating the resulting $\mathrm{CACE}$ estimate over the covariates as before.

\section{Simulation Study}

\subsection{Description of the Study}

In the simulation, one binary baseline covariate was considered. Values of $r_{i}, c_{i}, b_{i}$, and $y_{i}$ were generated as follows, $\left[r_{i}\right] \sim$
$\operatorname{Bernoulli}\left(p_{R}\right),\left[c_{i}, b_{i} \mid r_{i}\right] \sim \operatorname{Multinomial}\left(\alpha_{C B}\right), \quad\left[y_{i} \mid b_{i}, c_{i}, r_{i}\right] \sim$ $F(\cdot)+0.5-0.5 c_{i}-c_{i} r_{i}-0.5 b_{i}+c_{i} b_{i}$. To resemble the JOBS II data, we choose $\left(\alpha_{00}, \alpha_{10}, \alpha_{01}, \alpha_{11}\right)=(0.3,0.35,0.15$, $0.20)$ and $p_{R}=0.65$. To test the sensitivity of the normality assumption of $y_{i}$, three distributions of $F(\cdot)$ are chosen: $N(0,1)$, $T(d f=4)$, and $\Gamma($ shape $=5$, scale $=0.2)$. The random numbers from $F(\cdot)$ are recentered and rescaled so that they have mean 0 and variance 1 .

Let $m_{i}^{B}$ or $m_{i}^{Y}$ be the missing data indicators for $b_{i}$ or $y_{i}$, respectively. We assume that $m_{i}^{B}$ and $m_{i}^{Y}$ are independent with each other. Four missing-data mechanisms are considered: two of them (MAR1 and MAR2) are ignorable, while the other two (LMAR and NMAR) are nonignorable. Specifically,

(1) Missing at random (MAR1), where the probability of missingness depends on observed values in the data set, and the missing-data mechanism satisfies the latent ignorability conditions and the compound exclusion restriction in Frangakis and Rubin (1999): $\operatorname{Pr}\left(m_{i}^{Y}=1 \mid c_{i}\right.$, $\left.r_{i}, b_{i}, y_{i}\right)=\operatorname{Pr}\left(m_{i}^{B}=1 \mid c_{i}, r_{i}, b_{i}, y_{i}\right)=\Phi\left(\alpha_{0}+\alpha_{C R} c_{i} r_{i}\right)$, where $\Phi(\cdot)$ is the cumulative standard normal probability function.

(2) Missing at random (MAR2), where the probability of missingness only depends on the observed values in the data set, and the missing-data mechanism satisfies the latent ignorability conditions but not the compound exclusion restriction in Frangakis and Rubin (1999): $\operatorname{Pr}\left(m_{i}^{Y}=1 \mid c_{i}, r_{i}, b_{i}, y_{i}\right)=\operatorname{Pr}\left(m_{i}^{B}=1 \mid c_{i}, r_{i}, b_{i}, y_{i}\right)=$ $\Phi\left(\alpha_{0}+\alpha_{R} r_{i}+\alpha_{C R} c_{i} r_{i}\right)$. Hence $\operatorname{Pr}\left(m_{i}^{Y}=1 \mid c_{i}=0, r_{i}=\right.$ $\left.0, b_{i}, y_{i}\right)=\Phi\left(\alpha_{0}\right) \neq \operatorname{Pr}\left(m_{i}^{Y}=1 \mid c_{i}=0, r_{i}=1, b_{i}, y_{i}\right)=$ $\Phi\left(\alpha_{0}+\alpha_{R} r_{i}\right)$.

(3) Latent missing at random (LMAR) (Frangakis and Rubin, 1999), where the probability that $y_{i}$ is missing depends upon the compliance status $c_{i}$ which is unobservable for the control group subjects. In addition, the conditional distribution $\left(y_{i}, m_{i} \mid c_{i}, r_{i}\right)$ for never-takers satisfies the CER assumption. The probability that $b_{i}$ is missing depends only on $r_{i}$ : $\left[y_{i}, m_{i}^{Y} \mid c_{i}=0, r_{i}=0, b_{i}\right]=$ $\left[y_{i}, m_{i}^{Y} \mid c_{i}=0, r_{i}=1, b_{i}\right], \operatorname{Pr}\left(m_{i}^{Y}=1 \mid c_{i}, r_{i}, b_{i}, y_{i}\right)=$ $\Phi\left(\alpha_{0}+\alpha_{C} c_{i}+\alpha_{C R} c_{i} r_{i}\right)$, and $\operatorname{Pr}\left(m_{i}^{B}=1 \mid c_{i}, r_{i}, b_{i}, y_{i}\right)=$ $\Phi\left(\alpha_{0}+\alpha_{R} r_{i}\right)$.

(4) Not missing at random (NMAR), where the probability that $y_{i}$ is missing depends upon values of baseline covariate $b_{i}$, which contain missing values: $\operatorname{Pr}\left(m_{i}^{Y}=1 \mid c_{i}\right.$, $\left.r_{i}, b_{i}, y_{i}\right)=\operatorname{Pr}\left(m_{i}^{B}=1 \mid c_{i}, r_{i}, b_{i}, y_{i}\right)=\Phi\left(\alpha_{0}+\alpha_{X} b_{i}\right)$.

For each of 250 generated data sets, the following methods are applied for causal inferences: $I V n$ and $I V$ are the naive IV and the IV estimators defined in Section 2.4, and $I V n L$ and $I V L$ are the corresponding extended IV estimators assuming latent ignorable mechanisms. For likelihood-based methods, estimates of the CACE as well as other model parameters are obtained based on extended general location models. Specifically, MLBD (for maximum likelihood before deletion) is the ML estimate of the CACE of the data before generating missing values in $b_{i}$ and $y_{i}$; MLI and BYSI are the ML and Bayesian estimates of the CACE assuming ignorable mechanisms; MLLI and BYSLI are the ML and Bayesian estimates of the CACE assuming latent ignorable mechanisms; and MLCC and BYSCC are the ML and Bayesian (posterior 
mean) estimates of the CACE of the data after deleting observations with missing values in $b_{i}$ or $y_{i}$.

\subsection{Results}

Simulation results of the CACE parameter are summarized in Tables 1 and 2. For each simulation condition, biases and root mean square errors (RMSE) of the estimates of the CACE from each method are reported, after being multiplied by 1000. In addition, confidence intervals for each parameter are constructed from normal approximations based on estimates for that parameter, specifically for the $i$ th parameter, the $100 P \%$ confidence interval is constructed as $E S T_{i} \pm Z_{P}$. $S E_{i}$, for $P=0.5,0.6,0.8,0.9, E S T_{i}$ and $S E_{i}$ are the sample mean and standard error of estimates for the $i$ th parameter, and $Z_{P}$ is the $\{100(1-P) / 2\}$ th quantile of the standard normal distribution. Standard errors are calculated as standard deviations from the Gibbs draws for likelihood-based methods and as standard deviations from 200 bootstrap sample estimates for instrumental variable methods. Coverage probabilities of the confidence intervals of parameters are then obtained and compared.

As expected, estimates from the MLBD are the best, with the smallest RMSE, and estimates from $I V_{n}$ and $I V_{n} L$ are the worst with the largest biases and RMSEs. Naive IV methods assume a constant compliance rate for different values $B$ and this is not consistent with the data-generating process. Both the IV method and the likelihood-based methods are robust for the CACE estimates based on these simulation results. We observe no evidence that the IV methods are more robust to model misspecification than the likelihood-based methods. The RMSEs from IV are slightly larger than those from the likelihood-based methods. Probability coverages are good and consistent with the corresponding point estimates in general.

For the likelihood-based methods, ML estimates agree closely with the Bayesian estimates. RMSRs from the MLCC and BYSCC estimates are larger than the RMSEs from MLI and BYSI estimates. Also, estimates from models with the correct mechanisms perform better than those with incorrect mechanisms. Specifically, they are estimates from MLI, BYSI, MLLI, and BYSLI under MAR1, from MLI and BYSI under MAR2, and from MLLI and BYSLI under LMAR. When the missing-data mechanism is NMAR, i.e., missingness depends upon $B$, estimates from MLCC and BYSCC show no significant biases for $N(0,1)$ and $\Gamma(5,0.2)$ cases.

For the $N(0,1)$ case, estimates from MLLI, BYSLI, BYSLI, and IVL, which assume latent ignorable mechanisms, have larger biases than those from MLI, BYSI, and IVI when the latent ignorability conditions are not satisfied. Estimates from MLLI, BYSLI, and IVL are significantly biased when the true underlying missing-data mechanism is NMAR.

\section{Application}

As described in Section 1, the JOBS II (Vinokur et al., 1995) intervention project developed at the University of Michigan was to test a preventive intervention for unemployed workers. In the JOBS (JOB Search) II intervention setting, respondents were recruited from four offices of the Michigan Employment Security Commission in southeastern Michigan. Based on the screening questionnaire $\left(T_{0}\right)$ data, only people

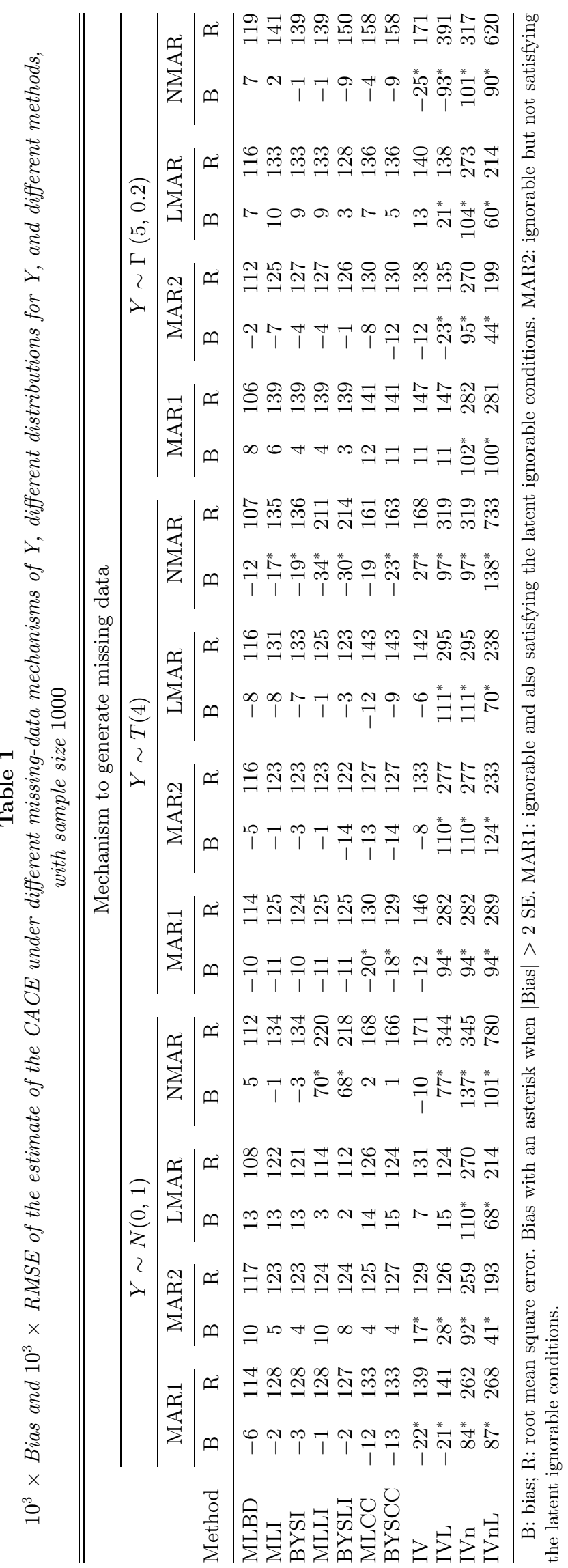


Table 2

Probability coverage of the estimate of the CACE under different missing-data mechanisms of $Y$, different distributions for $Y$, and different methods, with sample size 1000

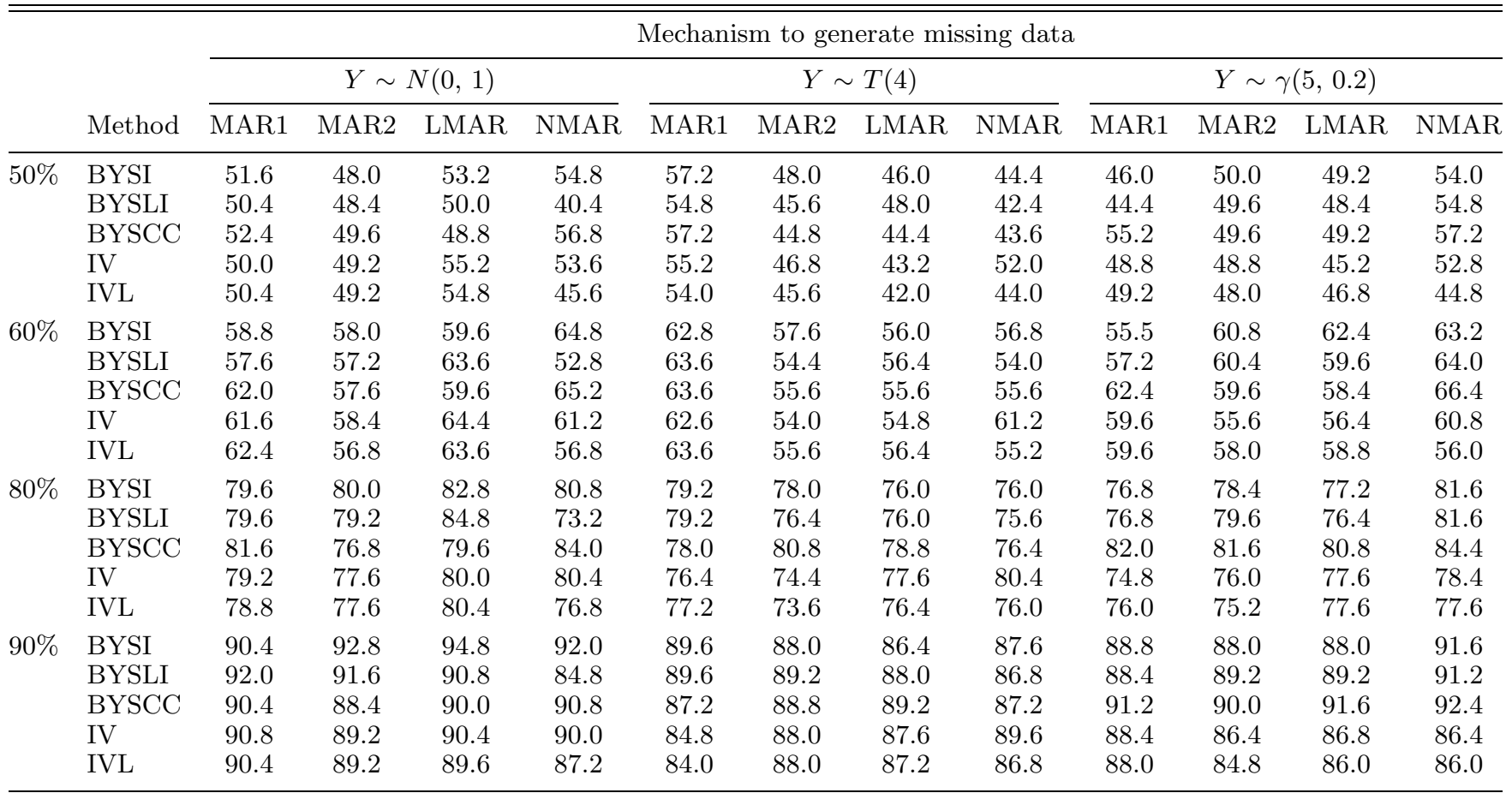

MAR1: ignorable and also satisfying the latent ignorable conditions. MAR2: ignorable but not satisfying the latent ignorable conditions.

who met the screening criteria were invited, randomized to the field study, and mailed pretest $\left(T_{1}\right)$ questionnaires.

A total of 1801 people returned their $T_{1}$ questionnaires and were enrolled in the study. Each subject in the control group was mailed a booklet describing job search methods and tips, and each subject in the experimental treatment group was mailed the same booklet and an invitation to participate in five 4-hour job search seminars. Follow-up questionnaires were mailed to all the respondents 2 months $\left(T_{2}\right)$ and 6 months $\left(T_{3}\right)$ after the week of the job search seminars. Measures of depression, financial strain, assertiveness, risk score, distress symptom, role and emotional functioning, job search self-efficacy, self-esteem, internal control orientation, mastery, intervention process, re-employment, as well as demographics variables were obtained through the questionnaires. The reemployment outcome at $T_{3}$ is the outcome of interest here, and it is binary.

Previous analyses (Vinokur and Price, 1995; Little and Yau, 1998) demonstrate that the intervention primarily benefited the re-employment and the mental health outcomes of respondents who are at high risk of experiencing depression in the future. The analysis focuses on data from the high-risk group, which includes 715 subjects. Because people who were randomized to the control group are not allowed to attend some of the seminars, there are only compliers and never-takers in the study.

Table 3 presents the summary statistics of the covariates measured at baseline for the high-risk group subjects, by the randomly assigned treatment $R$ and the compliance $C$ (for treatment group only, as $C$ is unknown for control group subjects). Comparison of the first two columns shows that the randomization did a fair job in balancing the covariate distributions across the two treatment groups.

To ensure enough participants in the high-risk group receiving the intervention, about two thirds of the high-risk group subjects (481 out of 715 ) were randomized to the treatment group. Among the 481 subjects in the treatment group, $58 \%$ (280 out of 481) of them attended at least one job search seminar and they are the compliers. The others did not attend any seminar and they are the never-takers. Table 3 shows that older subjects with more education, stronger motivation to attend the intervention, higher income, and lower assertiveness are more likely to be compliers.

Two models with exactly the same form are fitted, with different subjects included. While the "Partial" models include only those observations with no missing values in the baseline covariates, 600 subjects in the high-risk group, the "Full" models include all 715 subjects in the analysis. The covariates involved in the model fitting are: indicator of not married, indicator for nonwhite, age, school grade completed, motivation to attend, assertiveness, economic hardship, and attitude toward job search. Because of the normality assumption for the continuous variables in the extended general location models, logarithm transformations are applied to school grade completed.

Table 4 summarizes the estimation results of the probit models assuming ignorable missing-data mechanism, where the standard errors of the ML estimates are calculated from 
Table 3

Summary statistics of the covariates by treatment $R$ and compliance $C$, high-risk group

\begin{tabular}{lccrc}
\hline \hline & $\begin{array}{c}R=0 \\
\text { Mean (SD) } \\
\text { Covariate }\end{array}$ & $\begin{array}{c}R=1 \\
\text { Mean (SD) } \\
(n=481)\end{array}$ & $\begin{array}{c}R=1 \text { and } C=1 \\
\text { Mean (SD) } \\
(n=280)\end{array}$ & $\begin{array}{c}R=1 \text { and } C=0 \\
\text { Mean (SD) } \\
(n=201)\end{array}$ \\
\hline Age & $36.45(10.08)$ & $36.60(10.33)$ & $39.08(10.18)$ & $33.65(9.73)$ \\
School & $13.28(2.01)$ & $13.35(2.02)$ & $13.81(2.04)$ & $12.82(1.86)$ \\
Motivation & $5.32(0.80)$ & $5.31(0.82)$ & $5.43(0.82)$ & $5.16(0.79)$ \\
Income & $6.59(3.72)$ & $6.17(3.84)$ & $6.67(4.01)$ & $5.57(3.54)$ \\
Assertiveness & $3.05(0.87)$ & $3.11(0.92)$ & $3.01(0.92)$ & $3.23(0.92)$ \\
Not married & $0.58(0.49)$ & $0.65(0.48)$ & $0.66(0.48)$ & $0.64(0.48)$ \\
Economic hardship & $3.53(0.93)$ & $3.62(0.85)$ & $3.55(0.84)$ & $3.71(0.84)$ \\
Nonwhite & $0.21(0.41)$ & $0.22(0.41)$ & $0.18(0.39)$ & $0.25(0.44)$ \\
Attitude & $5.77(1.12)$ & $6.01(1.06)$ & $6.05(1.06)$ & $5.96(1.06)$ \\
Social undermine & $1.83(0.78)$ & $1.83(0.80)$ & $1.79(0.78)$ & $1.88(0.82)$ \\
Risk $\left(T_{0}\right)$ & $1.68(0.19)$ & $1.68(0.23)$ & $1.67(0.22)$ & $1.69(0.24)$ \\
\hline
\end{tabular}

250 bootstrap estimates. The $95 \%$ confidence intervals are estimated from the posterior distribution from the Gibbs draws, by the 2.5 th and the 97.5 th percentiles. The CACEs are the differences between the probability of being re-employed at $T_{3}$ for the compliers who participate in the job search seminars and the same probability for compliers who do not participate in the job search seminars. These are evaluated at the mean values of the continuous baseline covariates, and averaged over the distribution of the discrete covariates. Table 4 shows that the ML estimates and the corresponding Bayesian estimates are very similar for each model. Estimates from the the "Full" models are similar to those from the "Partial" model.

From the "Full" model that uses all the observations, the ML estimate of the probability of being re-employed at $T_{3}$ is increased by $16.1 \%$ by the intervention for a complier who has average education (grade 13) and the average score of attitude toward the job search. It is statistically significant with a $95 \%$ confidence interval of $(0.004,0.296)$. However, the ML estimate of the same CACE parameter from the "Partial" model is only $10 \%$ with a $95 \%$ confidence interval of $(-0.051,0.258)$ and it is not statistically significant. This difference is mainly due to the differences of the parameter estimates of the $C R$ term, which is closely related to the CACE parameter. The ML estimate of the coefficient for $C R$ from the "Full" model is 0.41 with a $95 \%$ confidence interval of $(0.010,0.764)$, while the estimate of the coefficient for $C R$ from the "Partial" model is reduced to 0.268 with a $95 \%$ confidence interval of $(-0.139$, 0.668). Two sources may contribute to the difference between the two estimates from the "Full" and the "Partial" models. First, more information is utilized in the "Full" model than in the "Partial" model and leads to smaller standard error of the

Table 4

Model fitting for the high-risk group with the outcome of re-employment at 6 months after the intervention, where "Full" indicates the results from data with all 715 observations, and "Partial" indicates the results from the data excluding those observations with missing values in covariates, 600 observations

\begin{tabular}{llrrr}
\hline \hline Parameter & & MLE $\pm \mathrm{SE}$ & \multicolumn{1}{c}{ Gibbs $\pm \mathrm{SE}$} & \multicolumn{1}{c}{$95 \mathrm{CI}^{* *}$} \\
\hline Intercept & (Full) & $-1.549 \pm 0.508$ & $-1.551 \pm 0.459$ & $(-2.491,-0.694)$ \\
& (Partial) & $-1.566 \pm 0.485$ & $-1.564 \pm 0.498$ & $(-2.530,-0.600)$ \\
$C$ & (Full) & $-0.446 \pm 0.245$ & $-0.406 \pm 0.224$ & $(-0.852,0.030)$ \\
$C R$ & (Partial) & $-0.233 \pm 0.267$ & $-0.230 \pm 0.235$ & $(-0.692,0.216)$ \\
& (Full) & $0.410 \pm 0.194$ & $0.379 \pm 0.194$ & $(0.010,0.764)$ \\
School & (Partial) & $0.268 \pm 0.226$ & $0.260 \pm 0.206$ & $(-0.139,0.668)$ \\
& (Full) & $0.079 \pm 0.031$ & $0.077 \pm 0.027$ & $(0.024,0.132)$ \\
Attitude & (Partial) & $0.086 \pm 0.030$ & $0.086 \pm 0.030$ & $(0.025,0.146)$ \\
& (Full) & $0.149 \pm 0.053$ & $0.151 \pm 0.051$ & $(0.052,0.251)$ \\
Nonwhite & (Partial) & $0.135 \pm 0.054$ & $0.136 \pm 0.054$ & $(0.033,0.243)$ \\
& (Full) & $-0.220 \pm 0.134$ & $-0.218 \pm 0.133$ & $(-0.479,0.038)$ \\
CACE* & (Partial) & $-0.249 \pm 0.167$ & $-0.253 \pm 0.148$ & $(-0.546,0.045)$ \\
& (Full) & $0.161 \pm 0.075$ & $0.148 \pm 0.075$ & $(0.004,0.296)$ \\
& (Partial) & $0.104 \pm 0.086$ & $0.100 \pm 0.079$ & $(-0.051,0.258)$ \\
\hline
\end{tabular}

${ }^{*} \mathrm{CACE}$ is calculated at the mean values of the school grade completed and the score of attitude toward the job search, then averaged over the distribution of nonwhite.

${ }^{* *} 95 \%$ CIs are calculated based on the 2.5th and 97.5th percentiles of the Gibbs draws. 
estimate. This is demonstrated by both the ML inference and the Bayesian inference. Second, the differences are also determined by the true underlying missing-data mechanism of the baseline covariates. Our model assumes that the mechanism is ignorable.

In addition, white people with more education and a higher score of attitude toward job search have better chances to be re-employed for both compliers and noncompliers. All 95\% confidence intervals for the regression parameters for School and Attitude toward job search do not include zero from both "Full" and "Partial" models. The effects of education and attitude toward the job search are statistically significant at a $95 \%$ confidence level.

\section{Conclusions and Discussion}

We have proposed an extended general location model to make inference for the CACE for data subject to noncompliance. The EGL model allows observations with missing values in the outcome or the baseline covariates to be included in the analysis, and it yields the same conditional distribution for the outcome and compliance conditioning on randomization and covariates as in Little and Yau (1998), thus generalizing that analysis. Models were presented for both ignorable and latent ignorable missing-data mechanisms, together with EM and Gibbs algorithms for computation. Simulations comparing the proposed methods with instrumental variable methods suggest that the IV methods and likelihoodbased methods are reasonably robust to certain departures from the model assumptions, and the likelihood-based methods are more efficient than the IV methods. An application to the JOBS II data with a binary re-employment outcome was presented.

Bloom's naive method (1984), which assumes constant compliance rate across different values of the covariates, may provide very biased results when compliance varies across covariates. Also, estimates from our LIMD model with the compound exclusion restriction did well when its assumptions were satisfied, but yielded estimates that were more biased than estimates from the IMD model for the nonignorable missing-data mechanism, where missingness of the outcome depended on a partially observed covariate. However, we emphasize that this finding is limited to our particular choice of LIMD model and simulation conditions. We think the LIMD assumption is likely to be more realistic than IMD in many settings, so other models based on this assumption are worthy of further study.

In this article we focused on a single outcome. The EGL model is fairly readily generalized to repeated measures normal data, by replacing the normal distribution of equation (3) by a multivariate normal with covariance structure suited to the repeated measures. Repeated binary outcomes can be accommodated by treating the outcomes in a normal model as latent thresholds, as we have done in the univariate case. Compliance is assumed all-or-none here. However, partial compliance is possible in practice. For example, in the JOBS II intervention trial, the treatment includes five job search seminars and some participants only receive part of them. In a clinical trial, patients may take only a fraction of the intended dosage of medications because of side effects. Thus models that allow for partial compliance are also worthy of future study.
Since our methods are based on parametric models, issues of model misspecification arise, and models that make weaker assumptions are worthy of consideration. Since compliance is treated here as a missing covariate and other missing covariate data are allowed, methods for fitting semiparametric models with missing covariates (Robins, Rotnitsky, and Zhao, 1994) could be applied to the problem, and compared with our parametric approach.

Details of the EM algorithm for the general location model and codes in Splus can be found at Little's web page (http:// www.sph.umich.edu/ rlittle/).

\section{ACKNOWLEDGEMENTS}

We thank the associate editor and referees for the useful comments on an earlier draft of the paper. This research was supported by NIMH grant P30 MH38330-12 and NSF grant DMS9803720.

\section{RÉSUMÉ}

La noncompliance est un problème fréquent dans les expériences avec attribution randomisée des traitements et les analyses standards reposant sur l'intention-de-traiter ou le traitement reçu ont des limitations. Une alternative attractive est d'estimer le Complier-Average Causal Effect (CACE), qui est l'effet moyen du traitement pour la sous-population des sujets qui seraient compliants à l'un ou l'autre des traitements (Angrist, Imbens and Rubin, 1996, ci-après AIR). Nous proposons une extension du modèle général de position afin d'estimer le CACE à partir de données avec noncompliance et données manquantes pour le critère d'évaluation et les covariables. Des modèles sont développés pour des critères continus ou catégoriels et des mécanismes de données manquantes ignorables ou latentes ignorables (Frangakis et Rubin, 1999). Les inférences pour ces modèles reposent sur l'algorithme EM et les méthodes bayésiennes MCMC. Nous présentons les résultats de simulations qui étudient la sensibilité aux hypothèses du modèle et l'influence du mécanisme des données manquantes. Nous appliquons également la méthode aux données d'une intervention pour la recherche d'emploi chez des chômeurs.

\section{REFERENCES}

Angrist, J. D., Imbens, G. W., and Rubin, D. B. (1996). Identification of causal effects using instrumental variables. Journal of American Statistical Association 91, 444-472.

Baker, S. G. (1998). Analysis of survival data from a randomized trial with all-or-none compliance: Estimating the cost-effectiveness of a cancer screening program. Journal of the American Statistical Association 93, 929934.

Barnard, J., Frangakis, C. E., Hill, J., and Rubin, D. B. (2002). School choices in NY city: Bayesian analysis of an imperfect randomized experiment (with discussion). In Case Studies in Bayesian Statistics, Volume 5, R. Kass, B. Carlin, A. Carriquiry, A. Gelman, I. Verdinelli, and M. West (eds), 33-97. New York: Springer-Verlag.

Bloom, H. S. (1984). Accounting for no-shows in experimental evaluation designs. Evaluation Review 8, 225-246.

Dempster, A., Laird, N., and Rubin, D. B. (1977). Maximum likelihood estimation from incomplete data using the EM 
algorithm. Journal of the Royal Statistical Society, Series B 39, 1-38.

Frangakis, C. E. and Rubin, D. B. (1999). Addressing complications of intent-to-treat analysis in the combined presence of all-or-none treatment-noncompliance and subsequent missing outcomes. Biometrika 86, 365-379.

Frangakis, C. E. and Rubin, D. B. (2002). Principle stratification in causal inference. Biometrics 58, 21-29.

Frangakis, C. E., Rubin, D. B., and Zhou, X. H. (2002). Clustered encouragement designs with individual noncompliance: Bayesian inference with randomization, and application to advance directive forms (with discussion and rejoinder). Biostatistics 3, 147-177.

Gelman, A. and Rubin, D. B. (1992). Inference from iterative simulation using multiple sequences (with discussion). Statistical Science 7, 457-472.

Goetghebeur, E. and Shapiro, S. (1996). Non-compliance, ethical imperative or mission impossible. Statistics in Medicine 15, 2813-2826.

Heckman, J. and Hotz, V. J. (1989). Choosing among alternative non-experimental method for estimating the impact of social programs: The case of manpower training. Journal of American Statistical Association 84, 862881.

Imbens, G. W. and Rubin, D. B. (1996). Bayesian inference for causal effects in randomized experiments with noncompliance. The Annals of Statistics 25, 305-327.

Imbens, G. W. and Rubin, D. B. (1997). Estimating outcome distributions for compliers in instrumental variables models. Review of Economic Studies 64, 555574 .

Little, R. J. A. and Rubin, D. B. (2002). Statistical Analysis with Missing Data, 2nd edition. New York: Wiley.

Little, R. J. A. and Yau, L. H. Y. (1998). Statistical techniques for analyzing data from prevention trials: Treatment of no-shows using Rubin's causal model. Psychological Methods 3, 147-159.

Mark, S. D. and Robins, J. M. (1993). Estimating the causal effect of smoking cessation in the presence of confounding factors using a rank preserving structural failure time model. Statistics in Medicine 12, 1605-1628.

Olkin, I. and Tate, R. F. (1961). Multivariate correlation models with mixed discrete and continuous variables. Annals of Mathematical Statistics 32, 448-465.

Peng, Y. (2001). Causal inference for data with noncompliance and missing values. Ph.D. Thesis, University of Michigan.

Robins, J. M. (1998). Correction for non-compliance in equivalence trials. Statistics in Medicine 17, 269-302.

Robins, J. M. and Tsiatis, A. A. (1991). Correcting for noncompliance in randomized trials using rank preserving structural failure time models. Communications in Statistics 20, 2609-2631.

Robins, J. M., Rotnitsky, A., and Zhao, L. P. (1994). Estimation of regression coefficients when some regressors are not always observed. Journal of the American Statistical Association 89, 846-866.

Rubin, D. R. (1976). Inference and missing data. Biometrika 63, 581-592.

Sommer, A. and Zeger, S. L. (1991). On estimating efficacy from clinical trials. Statistics in Medicine 10, 45-52.

Vinokur, A. D., Price, R. H., and Schul, Y. (1995). Impact of the JOBS intervention on unemployed workers varying in risk for depression. American Journal of Community Psychology 23, 39-74.

Yau, L. and Little, R. J. A. (2001). Inference for the complieraverage causal effect from longitudinal data subject to noncompliance and missing data, with application to a job training assessment for the unemployed. Journal of the American Statistical Association 96, 1232-1244.

Zeger, S. L. and Liang, K.-Y. (2001). Comment on "Compliance as an explanatory variable in clinical trials," by B. Efron and D. Feldman. Journal of the American Statistical Association 86, 18-19.

Received April 2002. Revised February 2004. Accepted February 2004. 